Article

\title{
Multi-Objective Function for SWIPT System by SADDE
}

\author{
Wei Chien ${ }^{1,2} \oplus$, Chien-Ching Chiu ${ }^{3, *} \mathbb{}$, Yu-Ting Cheng ${ }^{3}$, Wei-Lin Fang ${ }^{3}$ and Eng Hock Lim ${ }^{4}$ \\ 1 Department of School of Electric and Information Engineering, Beibu Gulf University, \\ Qinzhou 535011, China; air180@seed.net.tw \\ 2 Ningde QianWei Industry Technology Co., Ningde 352000, China \\ 3 Department of Electrical Engineering, Tamkang University, Tamsui 251301, Taiwan; \\ rainsstop@gmail.com (Y.-T.C.); tf0064419@gmail.com (W.-L.F.) \\ 4 Department of Electrical and Electronic Engineering, Universiti Tunku Abdul Rahman, Bandar Sungai Long, \\ Kajang, Selangor 43000, Malaysia; limeh@utar.edu.my \\ * Correspondence: chiu@ee.tku.edu.tw
}

Received: 13 March 2020; Accepted: 24 April 2020; Published: 30 April 2020

\begin{abstract}
Simultaneous wireless information and power transfer (SWIPT) optimization with multiple objective function optimization is presented in the millimeter band in this paper. Three different objective functions that are used for harvest power (HP), capacity, and bit error rate (BER) were studied. There are three different nodes in real environment for wireless power transfer (WPT) and SWIPT. The channel estimation calculated by shooting and bouncing ray/image techniques includes multi-path, fading effect, and path-loss in the real environment. We applied beamforming techniques at the transmitter to focus the transmitter energy in order to reduce the multi-path effect and adjust the length of the feed line on each array element in order to find the extremum of the objective functions by the self-adaptive dynamic differential evolution (SADDE) method. Numerical results showed that SWIPT node cannot achieve good performance by single objective function, but wireless power transfer (WPT) can. Nevertheless, both WPT and SWIPT nodes can meet the criteria by the multiple objective function. The harvesting power ratio as well as the BER and capacity can be improved by the multiple objective function to an acceptable level by only reducing a little harvesting energy compared to the best harvesting energy for the single objective function. Finally, the multiple optimization function cannot merely provide good information quality for SWIPT node but achieve good total harvesting power for WPT and SWIPT node as well.
\end{abstract}

Keywords: energy harvesting; multi-objective function; evolution algorithm; channel capacity; wireless powered communication network (WPCN), simultaneous wireless information and power transfer (SWIPT)

\section{Introduction}

We can recognize two practical architectures of wireless power transfer (WPT) in communication systems: wireless powered communication networks (WPCNs) [1-7] and simultaneous wireless information and power transfer (SWIPT) [8-16]. The first one describes the situation where a downlink signal shares power and the uplink signal is utilized to transfer information. On the contrary, the other one describes a situation where both information and power can be acquired from the downlink signal. When the enormous wireless nodes are deployed ubiquitously, WPT can be a convenient option for wireless nodes. However, some nodes not only need to be powered by a wireless system but also have information requests. The method for accomplishing this is known as SWIPT. The SWIPT method allows tools to gather power and transfer data simultaneously. 
In recent years, potential SWIPT applications have been investigated with the rapid development of mobile communication systems and the Internet of Things (IoT). The millimeter-wave SWIPT application is regarded as one of the best potential candidates for use in the future. There has been a wide array of millimeter-wave communications that can be used to support a wide bandwidth for applications with high data transmission and massive power accesses. Despite the potential of millimeter-wave application, there are many accompanying challenges that need to be investigated and solved. The high frequency will cause the path-loss and the shadowing effect in real environments. In particular, beamforming techniques using antenna arrays are suggested in order to conquer substantial depletion at a high regularity [17].

The work by Kamga and Aïssa [7] researched the efficiency of mmWave substantial multiple-input multiple-output WPCNs with and without rain depletion. A dynamic energy splitting receiver was recommended to split the obtained signal with flexible power proportion for power harvesting and also information decoding [8]. Zhang and Ho [9] examined a multiple-input multiple-output (MIMO) SWIPT system. Beamforming techniques were used for transmitting to the power and information receiver. In a study by $\mathrm{Ng}$ et al. [12], multi object source allocation for protected interaction in cognitive radio networks was researched. Sources and tasks scheduling for the SWIPT IoT systems while focusing on reducing the power usage needed to guarantee the minimal ordinary information rate and task performance rate were presented by Lee and Lee [13]. However, most papers deal with the optimal analytic solution with the signal to noise ratio (SNR) constraint for narrow band signal transmission. For wide band signal transmission, the bit error rate performance should be considered in the wireless wideband communication system due to the frequency selective effect of the multipath channel and inter-symbol interference (ISI) instead of SNR only. In this paper, a wideband circular array is used to manufacture the array pattern in order to optimize the bit error rate (BER), capacity, and the energy harvesting efficiency by evolution algorithms. To the best of our knowledge, the multiple objective function for this problem by self-adaptive dynamic differential evolution (SADDE) has not been previously reported in the literature.

The system model and academic formula are presented in Section 2, Section 3 explains the evolution algorithm. In Section 4, numerical results for four different criterion cases with evolution algorithm are presented and compared with the system quality and the harvesting power. Conclusions are presented in Section 5 .

\section{System Model}

An indoor millimeter wave system with WPT and SWIPT is considered for short distance. We utilize the ray-tracing method to compute the environment channel and the channel regularity feedback can be acquired as follows:

$$
H(f)=\sum_{i=1}^{N_{I M}} b_{i}(f) e^{j \psi_{i}(f)}
$$

where the frequency of sinusoidal wave is $f$ and the path index is $i$. Both $\psi_{i}(f)$ and $b_{i}(f)$ are the $i$-th phase shifts with receiving magnitudes for different frequencies. The total number of the receiving ray is $N_{I M}$. The regularity feedback can transform to the time domain by utilizing the inverted fast Fourier transform. We can compose the time domain impulse feedback of the equivalent baseband here as follows:

$$
\mathbf{h}(\mathbf{t})=\sum_{\mathbf{q}=1}^{\mathrm{Q}} \alpha_{\mathbf{q}} \delta\left(\mathbf{t}-\tau_{\mathbf{q}}\right)
$$

where we have the overall number of paths observed at time Q. $\alpha_{q}$ and $\tau_{q}$ are the channel gain and time delay for the $q$-th path, respectively.

Regarding the system nodes, there are three type nodes in our system, which is shown in Figure 1. First, there are the transmitter nodes. There are $\mathrm{N}$ antennas in the antenna array to protect against the path loss in the millimeter wave and to focus the transmission power efficiently. Second is the SWIPT 
node; this node uses one antenna to receive the signal information and harvest power. The third type of node is only used for harvesting power in downlink transmission.

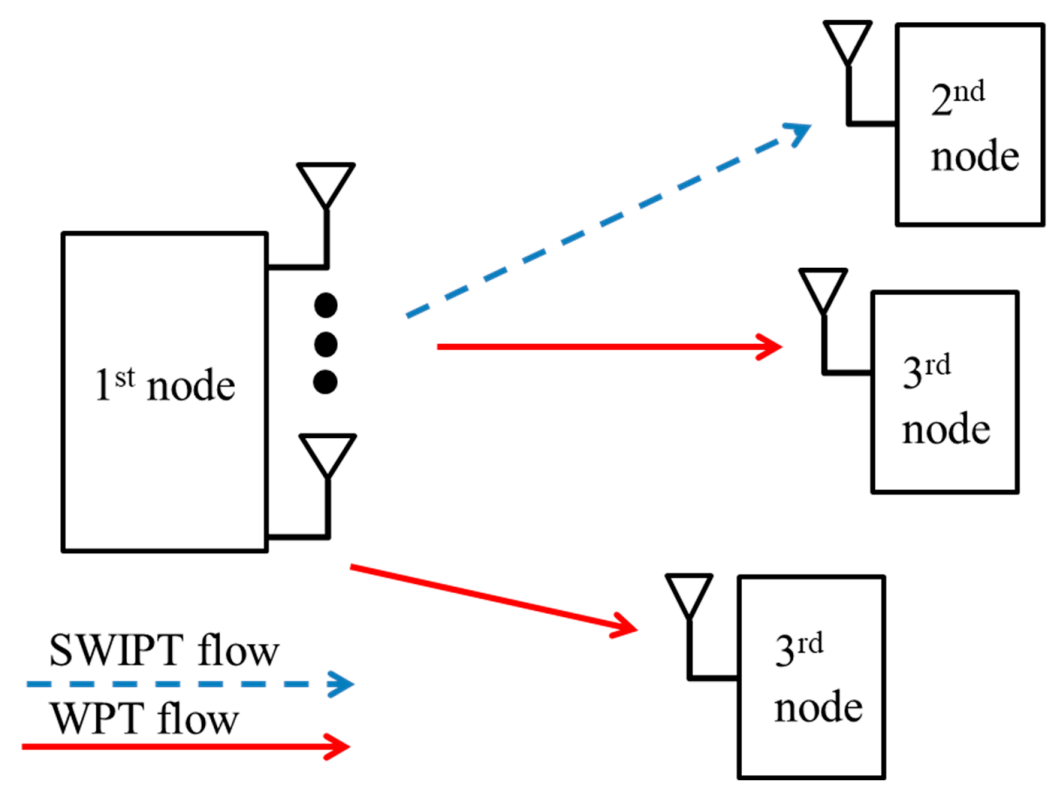

Figure 1. System node description. SWIPT: simultaneous wireless information and power transfer; WPT: wireless power transfer.

We made use of the power-splitting style in SWIPT node, as displayed in Figure 2. The received radio frequency $(\mathrm{RF})$ signals are divided into two streams for the information receiver and RF power harvester with various power levels.

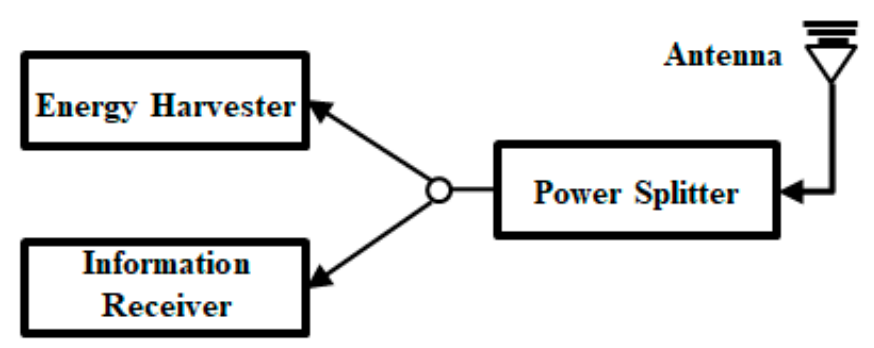

Figure 2. Receiver architecture for the SWIPT node.

A circular array with $\mathrm{N}$ millimeter wave dipole antennas is shown for the first type of node in Figure 3. 


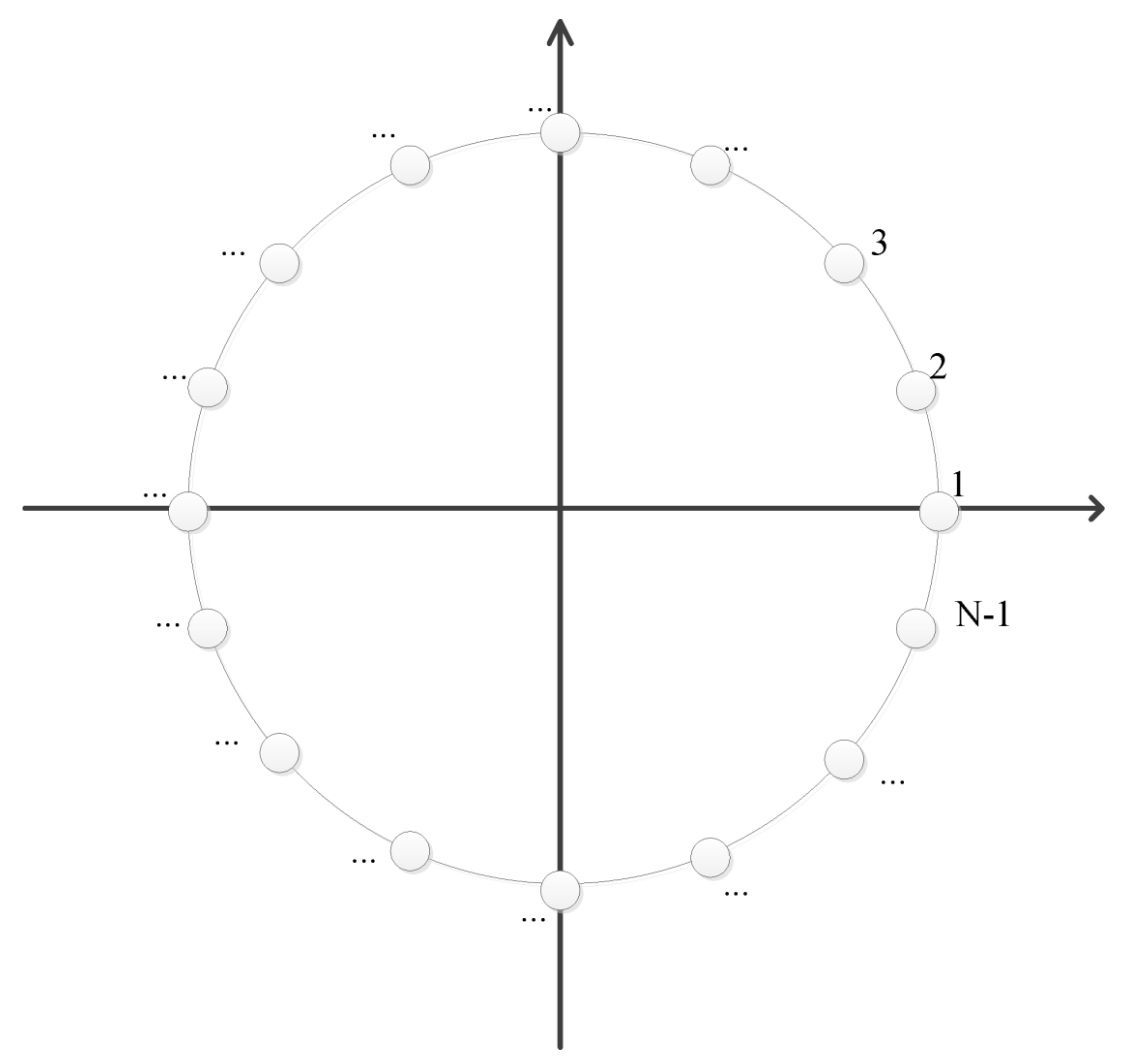

Figure 3. Deployment of the antenna array for the millimeter wave system.

The array factor for the antenna array is as follows:

$$
A F(\theta, \phi, f)=\sum_{n=1}^{N} W_{n} e^{j k a \sin \theta \cos \left(\phi-\frac{2 \pi}{N}(n-1)\right)}
$$

where the $\theta$ and $\boldsymbol{\phi}$ is the spherical coordinate system, $N$ is the total number of antennas in the circular array, and $\mathbf{a}$ is the distance from the antenna position to coordinate center. $W_{n}=I_{n} e^{j \alpha_{n}(f)}$ includes the excitation current $I_{n}$ and phase delay $\alpha_{n}(f) . k=2 \pi / \lambda$ is the wavenumber and $\lambda$ is the wavelength. $\alpha_{n}(f)$ can expressed as follows:

$$
\alpha_{n}(f)=-\frac{2 \pi f}{c} \sqrt{\varepsilon_{r}} \cdot \ell_{n}
$$

where $\varepsilon_{r}$ is the relative permittivity of the feed line, $\boldsymbol{\ell}_{\boldsymbol{n}}$ is the feed length of line needed to adjust the phase delay, and $c$ is the speed of light.

In the second type of node, the power-splitting architecture in the system is used, as shown in Figure 2. The obtained RF signals are divided into two streams for the information receiver and RF power collector with various power levels. Let $\eta \in[0, \mathbf{1}]$ signify the energy-dividing coefficient for the receiver (i.e., $\boldsymbol{\eta}$ is the portion of RF signals utilized for power gathering). The total energy collected from the receiver is modeled as:

$$
H P_{R}=\eta \int_{-\infty}^{\infty} h(t)^{2} d t
$$

In a SWIPT system, the quality of information is also important. Thus, we consider the BER and capacity as the information quality for the millimeter wave system. The BER considers the inter-symbol-interference in our case and is as follows [18]:

$$
B E R=\sum_{s=0}^{2^{s}} P\left(\vec{d}_{s}\right) \cdot \frac{1}{2} \operatorname{erfc}\left[\frac{V\left(t=s T_{d}\right)}{\sqrt{2} \sigma} \cdot\left(d_{s}\right)\right]
$$


where $\operatorname{erfc}(x)=\frac{2}{\sqrt{2}} \int_{x}^{\infty} e^{-y^{2}} d y$ is the complementary error function and $\left\{\vec{d}_{s}\right\}=\left\{d_{0}, d_{1}, \ldots d_{S}\right\}$ is the binary sequence.

The average channel capacity is as follows:

$$
C=\sum_{f=1}^{f=M_{F}} \frac{\Delta B \log \left(1+S N R_{R}(f)\right)}{B}(\text { bits } / \mathrm{sec} / \mathrm{Hz})
$$

where $C$ is the average channel capacity and $B$ is the bandwidth of the system. $\Delta B$ is the bandwidth for each different frequency channel. The signal to noise ratio at the receiver is $S N R_{R} \cdot M_{F}$ is the total number of frequencies for the system. The third type of node is used to harvest RF energy with full power in downlink transmission.

\section{Evolution Algorithm}

The flowchart of the SADDE algorithm is shown in Figure 4 [19,20]. In the first step, the algorithm initiates the parameter of population $X_{\mathbf{p}}^{\mathrm{g}}$ randomly. $\boldsymbol{p}$ is the number sequence in the total population. There are $d$ dimensional adjustment parameters in a population that are used to calculate the objective function. In the second step, the algorithm uses a mutation mechanism to get the vector $\mathbf{V}_{\mathbf{p}}^{\mathbf{g}+1}$. In the third step, the algorithm crossovers $\mathbf{V}_{\mathbf{p}}^{\mathbf{g}+1}$ with $\mathbf{X}_{\mathbf{p}}^{\mathbf{g}}$ to deliver vector $\mathbf{U}_{\mathbf{p}}^{\mathbf{g}+1}$. Finally, the algorithm compares $\mathbf{U}_{\mathbf{p}}^{\mathbf{g}+1}$ and $\mathbf{X}_{\mathbf{p}}^{\mathbf{g}}$ to update the $\mathbf{X}_{\mathbf{p}}^{\mathbf{g}+1}$ according to the objective value. All steps repeat until the termination conditions for the total generation number are reached.

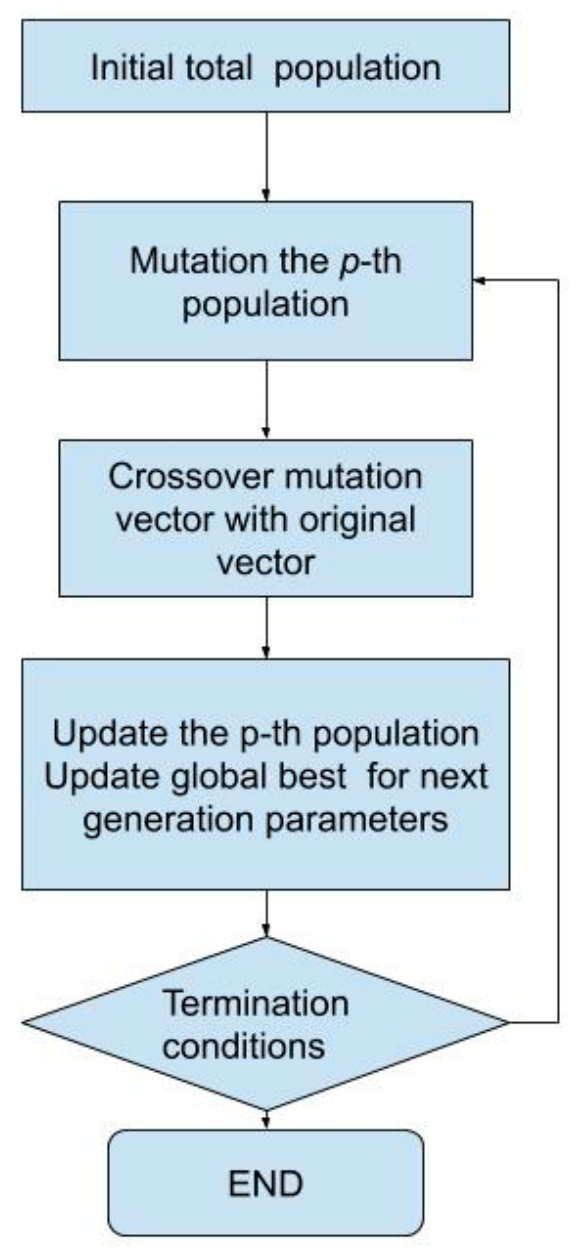

Figure 4. The self-adaptive dynamic differential evolution (SADDE) flow chart. 


\section{Numerical Result}

In this paper, a millimeter wave system from $59 \mathrm{GHz}$ to $60 \mathrm{GHz}$ is proposed, and the corresponding channel is calculated by ray tracing technology. The setting of the actual office environment is shown in Figure 5, and (Length, Width, Height) is used to describe the size of the furniture. There are three receivers ( $R \times 1, R \times 2, R \times 3)$ and one transmitter $(T x)$ in the office. We used short dipole antennas in this case. If the feed length of the transmitting antenna array is set to the same value, the harvesting powers at $R \times 1, R \times 2$, and $R \times 3$ are $2.75 \times 10^{-9}, 6.22 \times 10^{-9}$, and $6.16 \times 10^{-9}$, respectively. In our case, $I_{n}$ is set to 1 and the SADDE algorithm is utilized to adjust the feed length for $\alpha_{n}(f)$. We set the radius of the transmitting spherical antenna array a to $0.006 \mathrm{~m}$, while the separation distance between antennas is about $\frac{\lambda}{2}$ for decreasing the coupling in each antenna. The total number of the transmitting antennas is 16 for the beamforming technique. The $S N R_{T}$ is set to $40 \mathrm{~dB}$. Here, the $S N R_{T}$ is the transmission power to the noise ratio. The $\mathrm{Rx} 1$ is used to transfer the information and harvest power with the power-dividing architecture. We set $S N R_{T}$ to $37 \mathrm{~dB}$ at $\mathrm{Rx} 1$ for information decoding because the $\mathrm{Rx} 1$ uses $50 \%$ power-splitting for information decoding. Likewise, the $S N R_{T}$ for harvested power at $R \times 1$ is also $37 \mathrm{~dB}$ because the $50 \%$ power-splitting is $3 \mathrm{~dB}$. $\mathrm{R} \times 2$ and $\mathrm{R} \times 3$ are used to harvest power with the $S N R_{T}$ (The transmission power to the noise ratio) $40 \mathrm{~dB}$. In our study, there are four different objective functions, as shown in Table 1. Note that we use SADDE to find the minimum value of the objective features. The population size is set to 30 and the iteration is set to $500 . \mathrm{W}_{\mathrm{C}}, \mathrm{W}_{\mathrm{BER}}$, and $\mathrm{W}_{\mathrm{HP}}$ are the weighting to adjust the capacity, BER, and harvest power (HP), respectively, for the multiple objective function. The weighting is set according to the optimization of Rx 1 by SADDE with the corresponding single objective function. In other words, we use the algorithm to search the optimization for Rx1 with capacity ${ }_{\mathrm{RX} 1}, \mathrm{BER}_{\mathrm{RX} 1}$, and $\mathrm{HP}_{\mathrm{RX} 1}$, respectively. According to the numerical result, we can get the weighting of the multiple objective function. Thus, we set the weighting as $W_{\mathrm{BER}}=\frac{1}{0.11091401 \times 10^{-16}}$, $\mathrm{W}_{\mathrm{C}}=\frac{1}{0.86862899 \times 10^{-1}}$, and $\mathrm{W}_{\mathrm{HP}}=\frac{1}{0.23272111 \times 10^{-6}}$ for the multiple objective function. Note that the weighting considers the maximum value of optimization function for balancing multiple objectives.
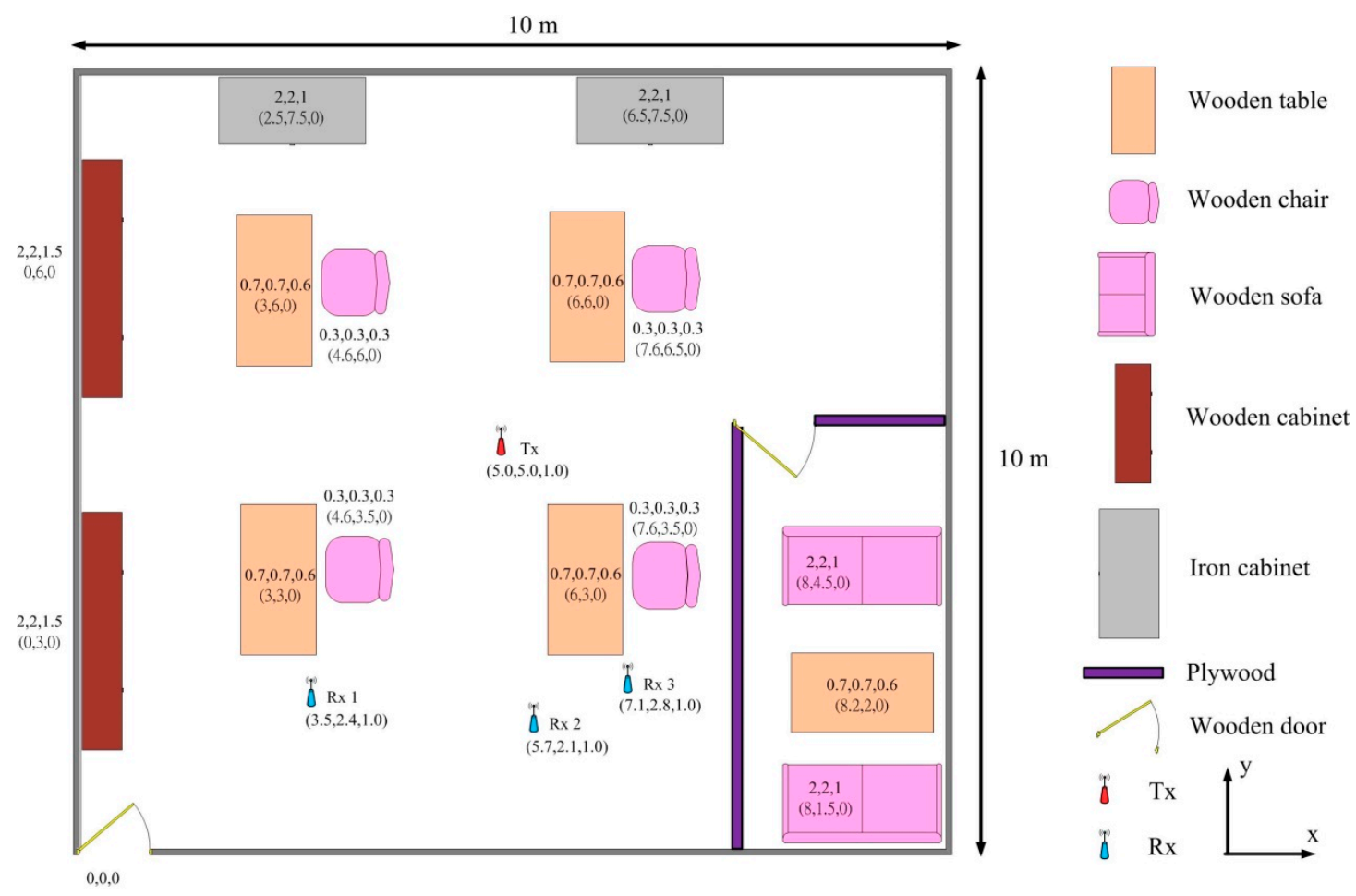

Figure 5. Layout of the office environment. 
Table 1. Objective functions for different cases. BER: bit error rate.

\begin{tabular}{cc}
\hline Objective Type & Objective Function \\
\hline BER & $=\mathrm{BER}_{\mathrm{R} \times 1}+\mathrm{BER}_{\mathrm{R} \times 2}+\mathrm{BER}_{\mathrm{R} \times 3}$ \\
Capacity & $=\frac{1}{\text { Capacity }_{\mathrm{R} \times 1}+\text { Capacity }_{\mathrm{R} \times 2}+\text { Capacity }_{\mathrm{R} \times 3}}$ \\
Harvesting Power & $=\frac{1}{\mathrm{HP}_{\mathrm{R} \times 1}+\mathrm{HP}_{\mathrm{R} \times 2}+\mathrm{HP}_{\mathrm{R} \times 3}}$ \\
Multiple Objective & $=\frac{1}{\mathrm{~W}_{\mathrm{C}} \times \text { Capacity }_{\mathrm{R} \times 1}+1 /\left(\mathrm{W}_{\mathrm{BER}} \times \mathrm{BER}_{\mathrm{R} \times 1}\right)+\mathrm{W}_{\mathrm{HP}} \times \mathrm{HP}_{\mathrm{Total}}}$ \\
\hline
\end{tabular}

Figure 6 shows the BER at Rx1 for different object functions. In Figure 6, we can see that the BERs for the multiple objective function and BER objective function are very low, at $1.52 \times 10^{-6}$ and $1.29 \times 10^{-6}$ at $R \times 1$, respectively, Note that the total BER sum of all receivers ( $R \times 1, R \times 2$, and $R \times 3$ ) are 1.9996 and $3.5583 \times 10^{-6}$ for the multipath objective function and BER objective function, respectively. Thus, if we compare the BER at all receivers in this case, the BER objective function is much smaller than the multiple objective function, since the BER objective function optimizes all receivers, but the multiple objective function did not consider the BER at Rx2 and Rx3.

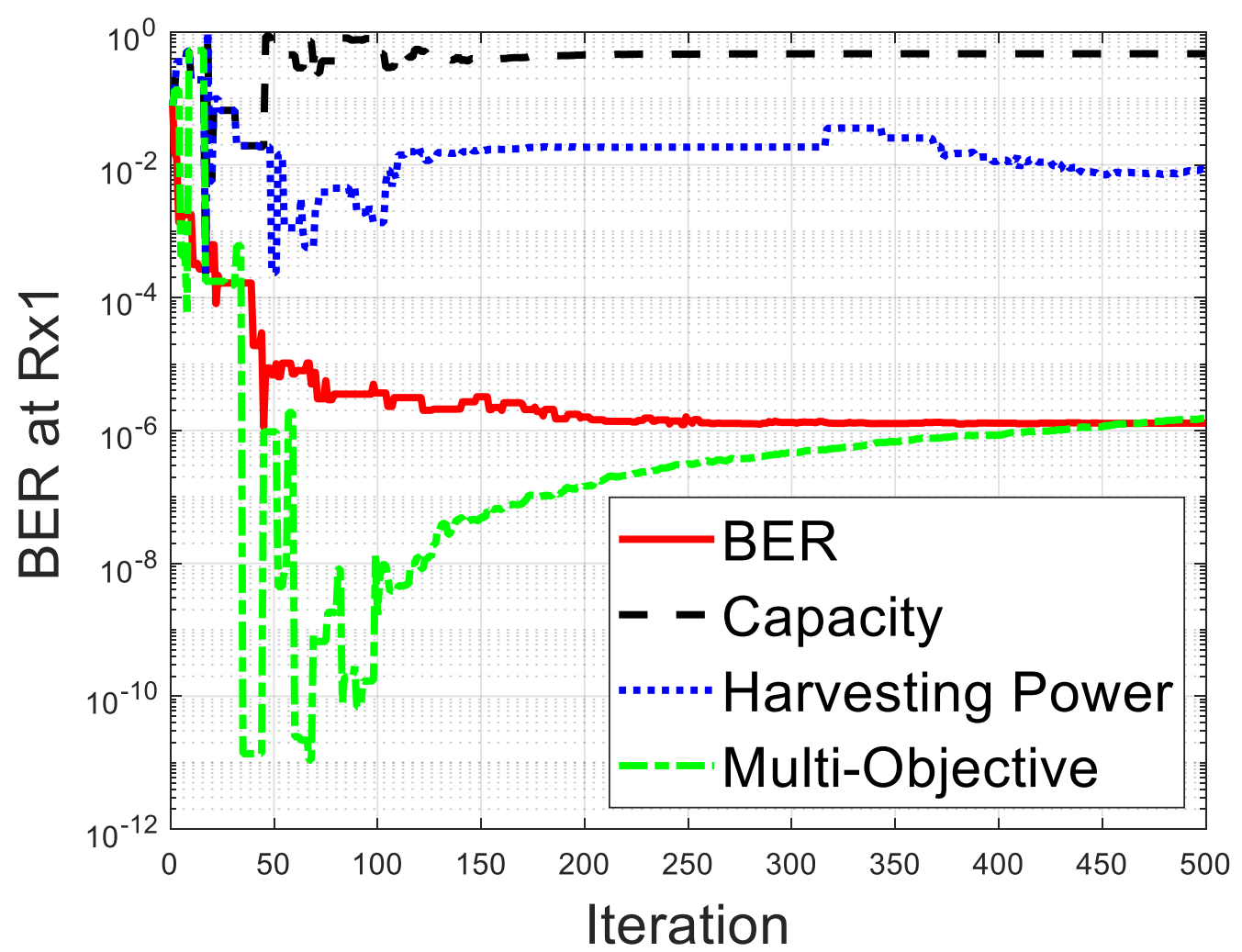

Figure 6. Comparison of different objective functions for BER at Rx1.

Figure 7 shows the values of capacity at Rx 1 for different object functions. The capacity at Rx 1 for the multiple objective function is higher than that for the capacity objective function. The capacity sums of all receivers are 0.2242 and 0.2102 for the capacity objective function and the multiple objective function, respectively. It is found that the capacity sums of all receivers are the largest for the capacity objective function. Note that the objective function not only considers Rx1's performance but also optimizes the other objectives. As a result, the optimization of Rx1 will not always approach to the extremum, since the objective function optimizes all objectives. The Rx1's capacity for harvesting power objective is the smallest, since the Rx1 uses the power-splitting architecture for SWIPT. As a result, the optimization focuses on optimizing $R \times 2$ and $R \times 3$, since the contribution of total harvesting power is large for $\mathrm{R} \times 2$ and $\mathrm{R} \times 3$ compared to $\mathrm{R} \times 1$. 


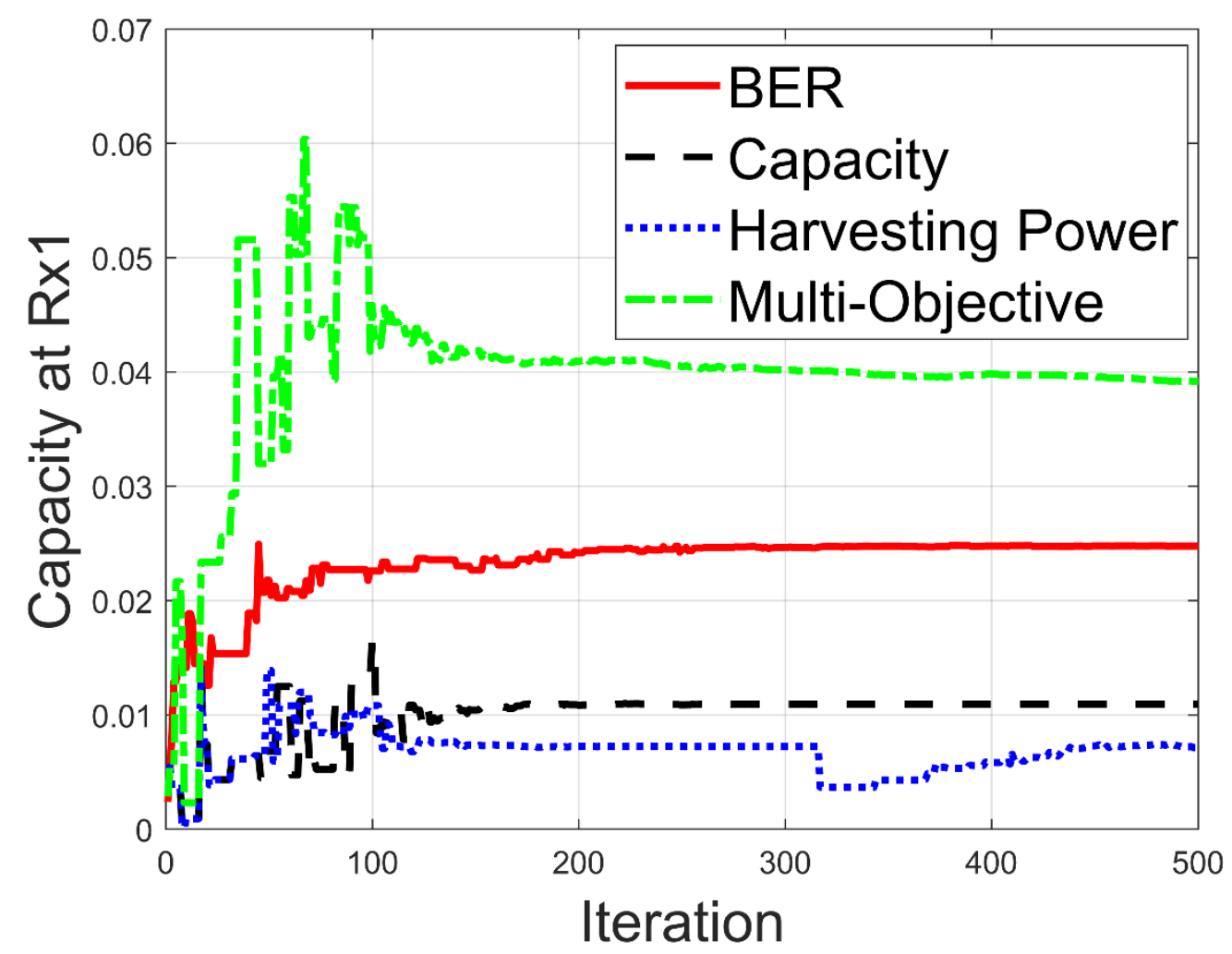

Figure 7. Comparison of different objective function for capacity at Rx1.

Figure 8 depicts the harvesting power for different objective functions. In Figure 8 , it can be seen that the total harvesting power for the HP objective function, the capacity object function, and the multiple objective function are larger than the BER objective functions. The total harvesting power ratio for the HP objective function and the capacity objective function are 101 and 104, respectively. The best harvesting power ratio is 112 for the multiple objective function. Although the harvesting power of the $\mathrm{HP}$ objective function and the capacity objective function are close to the multiple objective function, the BER at Rx1 is worse than for the multiple objective function. This means that the multiple objective function can consider all of the criteria at the same time. Figure 9 shows the harvesting power at $R \times 1$, $R \times 2$, and $R \times 3$ for the HP objective function. From Figure 9, it is clear that the harvesting power at $R \times 2$ and $R \times 3$ is larger than $R \times 1$. The harvesting powers at $R \times 1, R \times 2$, and $R \times 3$ are plotted in Figure 10 for the multiple objective function. The multiple objective function not only focuses on $R \times 2$ and $R \times 3$, but also considers the quality at Rx1. Compared to Figure 9, it is clear that the harvesting power for $R \times 1$ increased a lot for the multiple objective function. Thus, the multiple objective function can adjust the total goal better than the single objective function and achieve the overall best solution. 


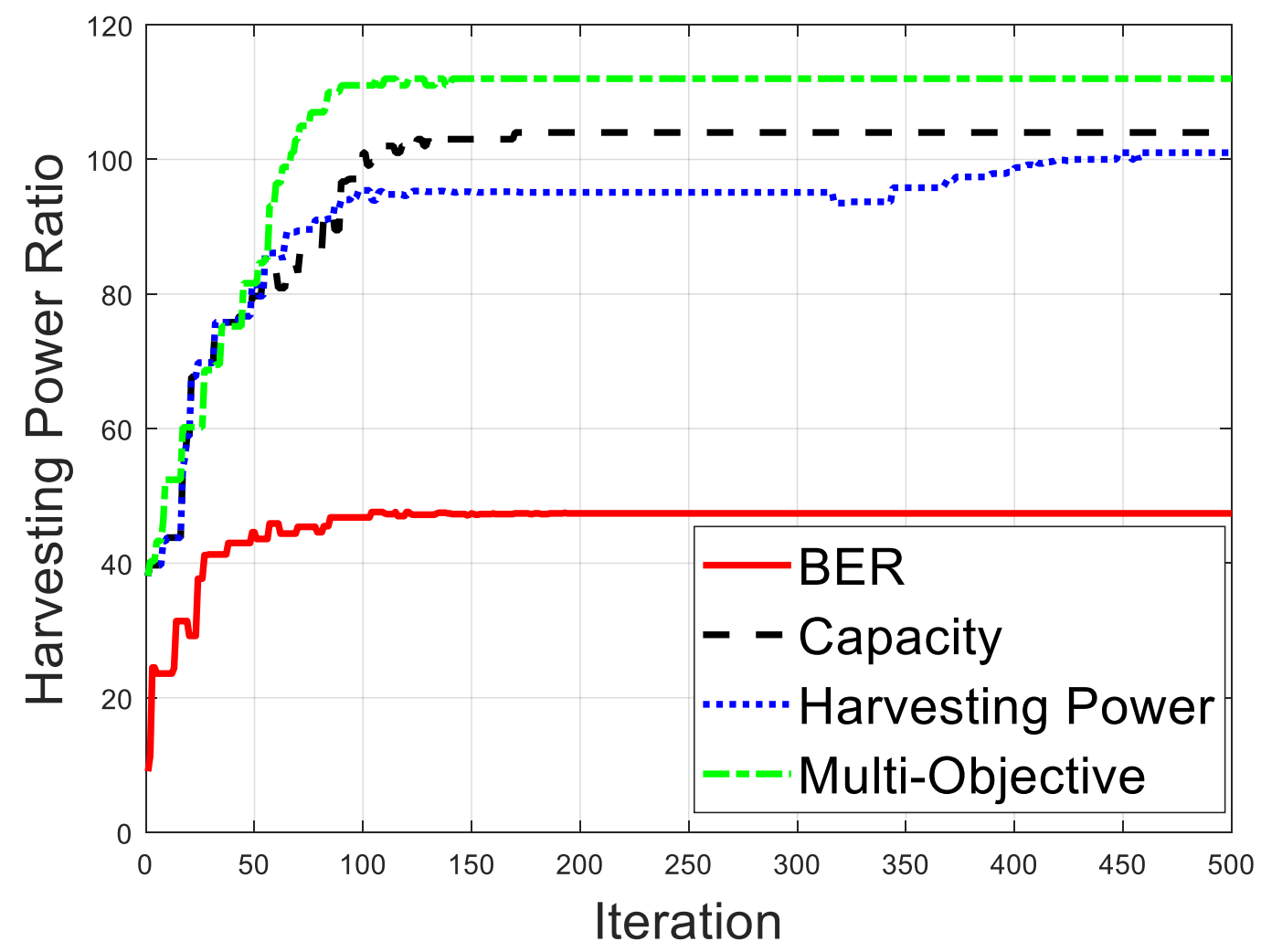

Figure 8. Harvesting power for different objective functions.

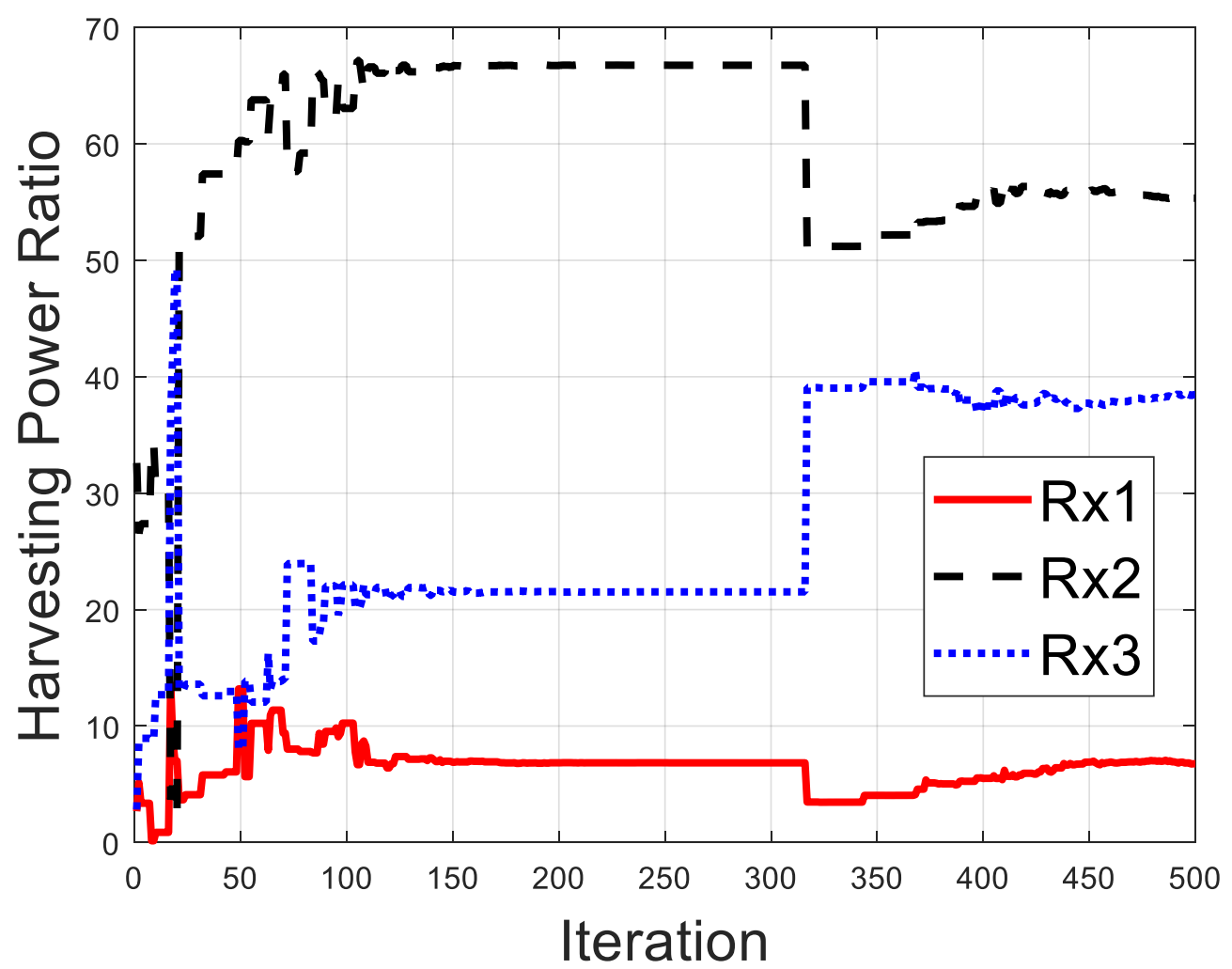

Figure 9. Harvesting power for the harvesting power (HP) objective function. 


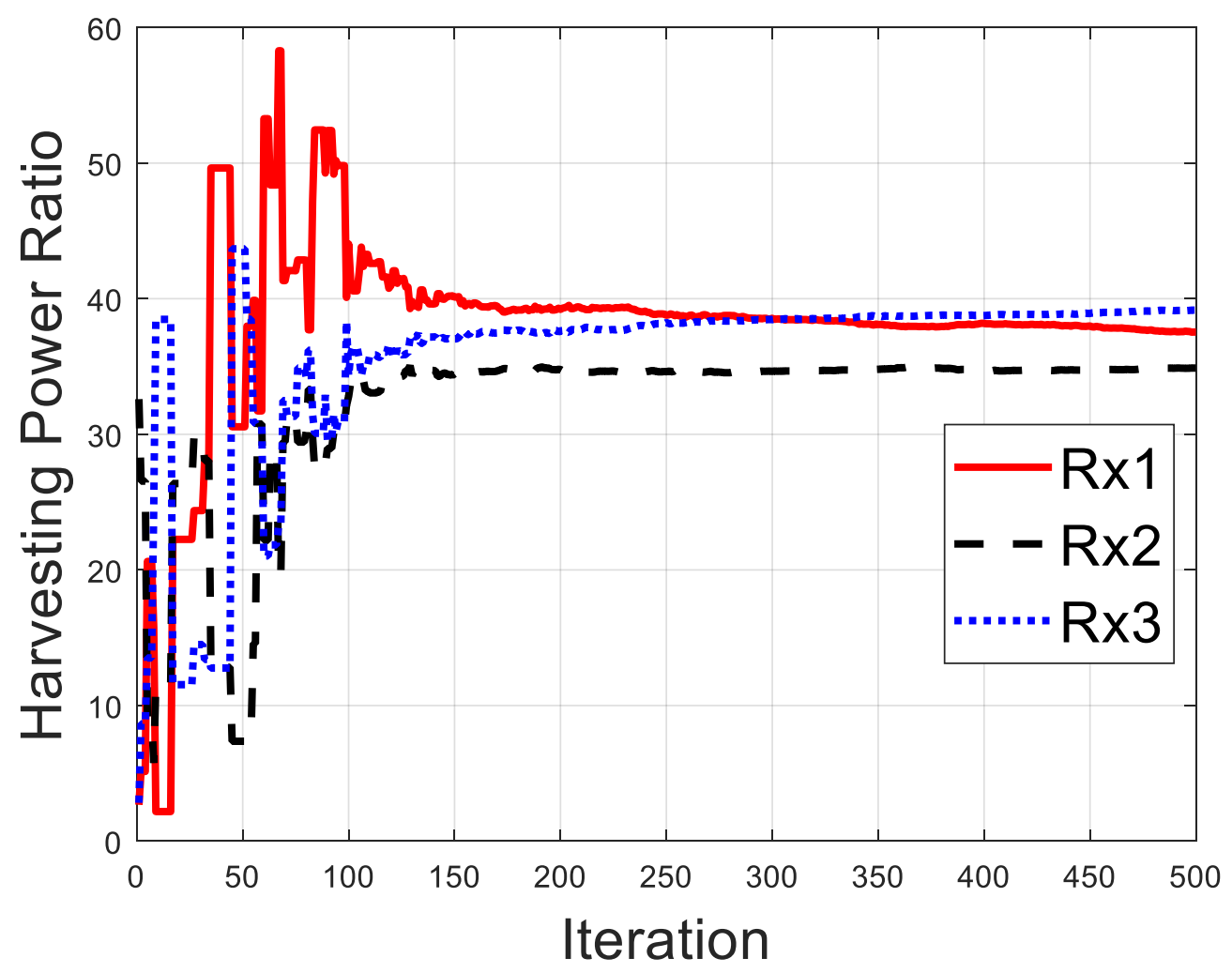

Figure 10. Harvesting power at $\mathrm{Rx} 1, \mathrm{R} \times 2$, and $\mathrm{R} \times 3$ for the multiple objective function.

Figures 11-14 show the radiation patterns for four different objective functions. In comparing Figures 5 and 11, the latter of which shows the location of the transmitter and the receivers, the beams for the multiple objective function and BER objective function have all directed to $R \times 1$, $R \times 2$, and $R \times 3$ with almost equal gain. However, for the BER objective function in Figure 12, it can be seen that the pattern for $R \times 1$ is the largest compared to those for $R \times 2$ and $R \times 3$. Because of the power splitting at $R \times 1$, the algorithm gives more power to lower the BER at Rx1 by high antenna gain. As a result, the total BERs of $R \times 1, R \times 2$, and $R \times 3$ can be reduced. Note that BER is not only affected by the SNR but is also affected by the multi-path with ISI. Since the BER objective function needs to minimize the sum of BER at $R \times 1, R \times 2$, and $R \times 3$, the pattern is not similar to that of the multiple objective function. The radiation patterns for the capacity objective function and HP objective function are plotted in Figures 13 and 14, respectively. In Figures 13 and 14, we can see the beam focus on Rx2 and Rx3. It is consistent with the result in Figure 7 in that the capacities at $R \times 1$ are small for the capacity objective function and $\mathrm{HP}$ objective function. According to these results, the single objective function can only successfully optimize BER, capacity, or power harvesting. However, the multiple objective function can balance the data quality and power harvesting when the suitable weighting is applied. 


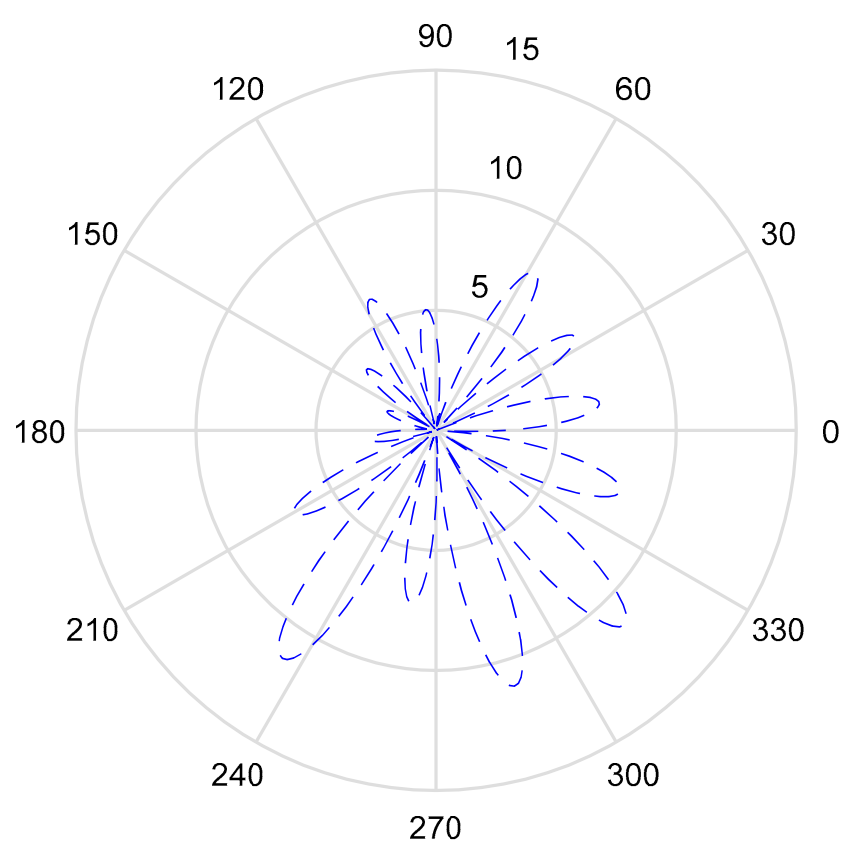

Figure 11. Radiation pattern for the multiple objective function.

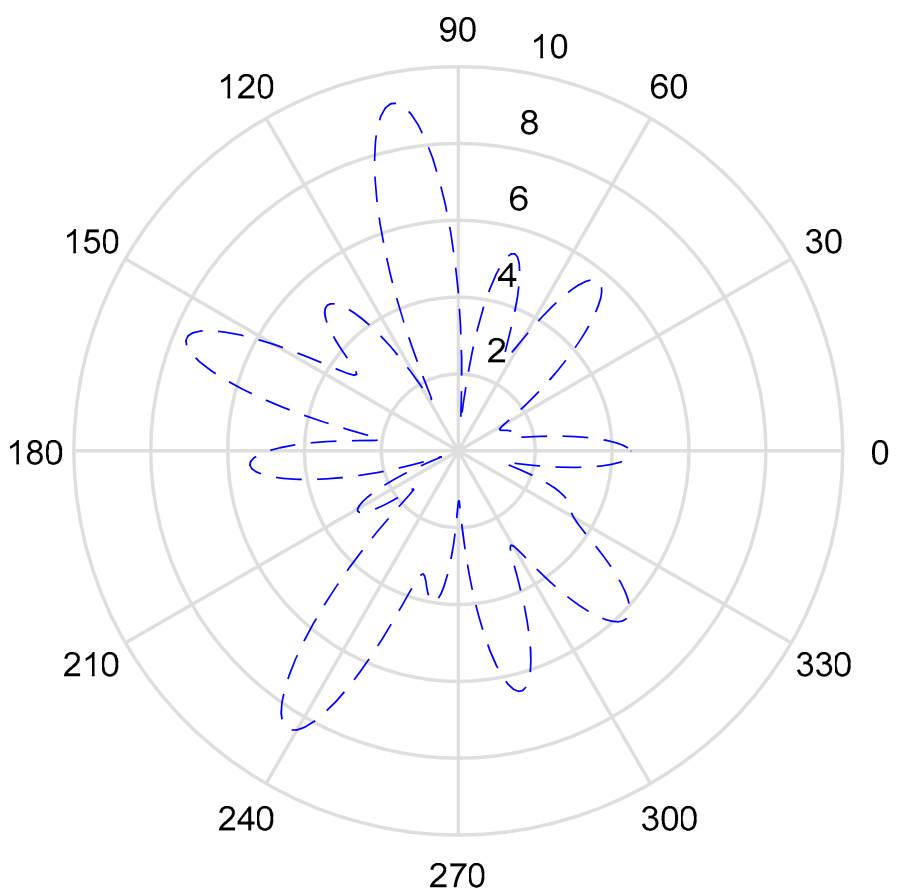

Figure 12. Radiation pattern for the BER objective function. 


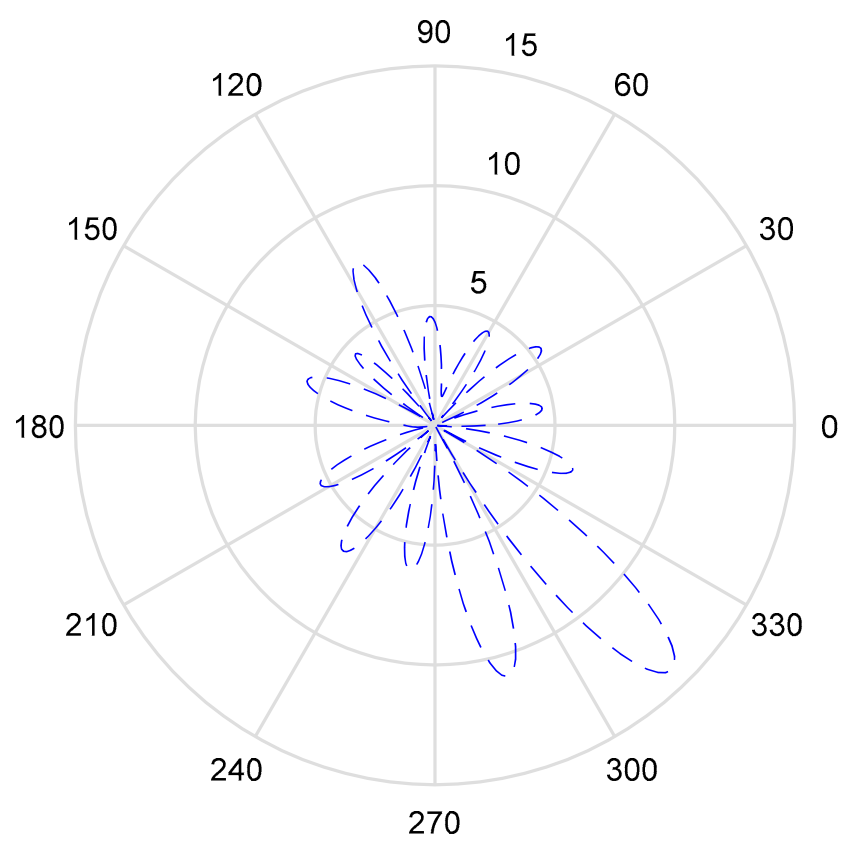

Figure 13. Radiation pattern for the capacity objective function.

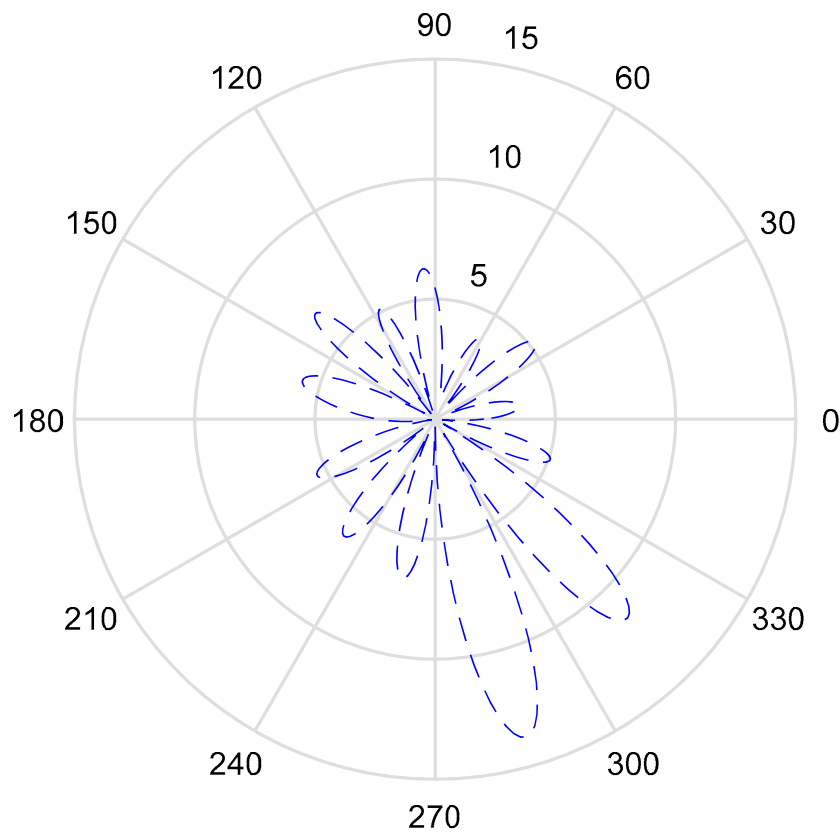

Figure 14. Radiation pattern for the HP objective function.

\section{Conclusions}

To optimize the harvesting power and information quality for WPT and SWIPT system at millimeter wave in a real environment, we compared different objective functions in this paper, and we used SADDE to optimize and compare the types of objective functions.

For the SWIPT system, we considered BER, capacity, and HP for harvesting power and information quality. The capacity objective function and the HP objective function were able to optimize all of the WPT type node receivers, but the information quality and the harvesting power demonstrated poor performance on the SWIPT type node. Although the information quality was able to optimize the best performance at SWIPT type node for the multiple objective function, the power harvesting was less than that for the HP objective function. The power splitting of Rx1 let the algorithm focus 
on the optimization of WPT type node for the HP objective function, since the HP objective function only considers the total receiving power from the transmitter instead of the information quality at the SWIPT type node. For these reasons, we used the multiple objective function to optimize the SWIPT node. Note that the BER was not only affected by SNR but also the inter-symbol-interference from multi-path. As a result, the multiple objective function shifted some harvesting power to improve the information quality at the SWIPT type node. Upon viewing the numerical results, we can see that the multiple objective function can balance the SWIPT type node and WPT type node. Thus, in the case of considering many criteria, we can use the multiple objective function to optimize our problem by suitable weighting.

Author Contributions: W.C., C.-C.C. and W.-L.F. contributed to the conception of the study. W.C., Y.-T.C. and W.-L.F. contributed significantly to analysis and manuscript preparation; W.C. and Y.-T.C. performed the data analyses and wrote the manuscript; C.-C.C. and Y.-T.C. helped perform the analysis with constructive discussions. All authors have read and agreed to the published version of the manuscript.

Funding: This research was funded by Research Initiation Project of Introducing High-level Talents from Beibu Gulf University of China, with grant number as 2017KYQD209.

Conflicts of Interest: The authors declare no conflict of interest.

\section{References}

1. Ju, H.; Zhang, R. Throughput maximization in wireless powered communication networks. IEEE Trans. Wirel. Commun. 2014, 13, 418-428. [CrossRef]

2. Tran, H.V.; Kaddoum, G.; Truong, K.T. Resource allocation in SWIPT networks under a nonlinear energy harvesting model: Power efficiency, user fairness, and channel nonreciprocity. IEEE Trans. Veh. Technol. 2018, 67, 8466-8480. [CrossRef]

3. Tran, H.V.; Kaddoum, G.; Abou-Rjeily, C. Collaborative RF and lightwave power transfer for next-generation wireless networks. IEEE Commun. Mag. 2020, 58, 27-33. [CrossRef]

4. Bi, S.; Zeng, Y.; Zhang, R. Wireless powered communication networks: An overview. IEEE Wirel. Commun. 2016, 23, 10-18. [CrossRef]

5. Psomas, C.; Krikidis, I. Backscatter communications for wireless powered sensor networks with collision resolution. IEEE Wirel. Commun. Lett. 2017, 6, 650-653. [CrossRef]

6. Psomas, C.; Krikidis, I. Energy beamforming wireless powered mmWave sensor networks. IEEE J. Sel. Areas Commun. 2019, 37, 424-438. [CrossRef]

7. Kamga, G.N.; Aïssa, S. Wireless power transfer in mmWave massive MIMO systems with/without rain attenuation. IEEE Trans. Commun. 2019, 67, 176-189. [CrossRef]

8. Zhou, X.; Zhang, R.; Ho, C.K. Wireless information and power transfer: Architecture design and rate-energy tradeoff. IEEE Trans. Commun. 2013, 61, 4754-4767. [CrossRef]

9. Zhang, R.; Ho, C.K. MIMO broadcasting for simultaneous wireless information and power transfer. IEEE Trans. Wirel. Commun. 2013, 12, 1989-2001. [CrossRef]

10. Boshkovska, E.; Ng, D.W.K.; Zlatanov, N.; Schober, R. Practical non-linear energy harvesting model and resource allocation for SWIPT systems. IEEE Commun. Lett. 2015, 19, 2082-2085. [CrossRef]

11. Pan, G.; Lei, H.; Yuan, Y.; Ding, Z. Performance analysis and optimization for SWIPT wireless sensor networks. IEEE Trans. Commun. 2017, 65, 2291-2302. [CrossRef]

12. Ng, D.W.K.; Lo, E.S.; Schober, R. Multiobjective resource allocation for secure communication in cognitive radio networks with wireless information and power transfer. IEEE Trans. Veh. Technol. 2016, 65, 3166-3184. [CrossRef]

13. Lee, H.S.; Lee, J.W. Resource and task scheduling for SWIPT IoT systems with renewable energy sources. IEEE Internet Things J. 2019, 6, 2729-2748. [CrossRef]

14. Shirichian, M.; Chamaani, S.; Akbarpour, A.; Del Galdo, G. Analysis and design of broadband simultaneous wireless information and power transfer (SWIPT) system considering rectifier effect. Energies 2018, 11, 2387. [CrossRef]

15. Masood, Z.; Jung, S.P.; Choi, Y. Energy-efficiency performance analysis and maximization using wireless energy harvesting in wireless sensor networks. Energies 2018, 11, 2917. [CrossRef] 
16. Liu, Y.; Xiong, K.; Zhang, Y.; Zhou, L.; Lin, F.; Liu, T. Multi-objective optimization of fog computing assisted wireless powered networks: Joint energy and time minimization. Electronics 2019, 8, 137. [CrossRef]

17. Roh, W.; Seol, J.Y.; Park, J.; Lee, B.; Lee, J.; Kim, Y.; Aryanfar, F. Millimeter-wave beamforming as an enabling technology for $5 \mathrm{G}$ cellular communications: Theoretical feasibility and prototype results. IEEE Commun. Mag. 2014, 52, 106-113. [CrossRef]

18. Chiu, C.C.; Chen, C.H.; Cheng, Y.T.; Lee, Y.L.; Chou, Y.K. Beamforming techniques at both transmitter and receiver for indoor wireless communication. J. Appl. Sci. Eng. 2018, 21, 407-412.

19. Chiu, C.C.; Cheng, Y.T.; Yang, C.H. Capacity optimization of multi-input/multi-output relay channel by SADDE algorithm. Ann. Telecommun. 2019, 74, 365-372. [CrossRef]

20. Chien, W.; Yu, C.Y.; Chiu, C.C.; Huang, P.H. Optimal location of the access points for MIMO-UWB systems. Appl. Sci. 2018, 8, 1509. [CrossRef]

(C) 2020 by the authors. Licensee MDPI, Basel, Switzerland. This article is an open access article distributed under the terms and conditions of the Creative Commons Attribution (CC BY) license (http://creativecommons.org/licenses/by/4.0/). 